\title{
The phase transitions character and microscopic models for $\mathrm{Sn}_{2} \mathrm{P}_{2} \mathrm{~S}(\mathrm{Se})_{6}$ ferroelectrics
}

\author{
Yu.Vysochanskii, A.Drobnich \\ Institute for Solid State Physics and Chemistry, \\ Uzhgorod National University, \\ 46 Pidgirna Str., 88000 Uzhgorod, Ukraine
}

Received August 30, 2002

The paper considers an example of concrete objects, i.e. ferroelectrics of $\mathrm{Sn}_{2} \mathrm{P}_{2} \mathrm{~S}_{6}$ family, for which, according to general estimations, a correlation between the shape of a phase diagram (the presence of a Lifshitz point and of a tricritical point) and a crossover "displacive to order/disorder" is observed. Simulations by means of microscopic models, which most adequately represent a dipole ordering in these crystals, are analyzed. A dimension dependence of a ferroelectric phase transition temperature for microcrystals of $\mathrm{Sn}_{2} \mathrm{P}_{2} \mathrm{~S}_{6}$ compound is considered.

Key words: ferroelectrics, displacive vs. order/disorder crossover, Lifshitz point, incommensurate phase, microscopic models, Monte Carlo simulations

PACS: $64.60 . \mathrm{Cn}$

\section{Introduction}

Structure phase transitions (PT) obviously are classified, from experimental point of view, as displacive type or order/disorder type. The observation of underdamped soft mode is considered to be an experimental evidence of a displacive type of transition, while in the case of order/disorder transition the transformation occurs from the equal probable distribution of some atoms between several positions to a more ordered configuration with a similar set of atomic positions. The self-consistent phonon approximation, or pseudo-spin models, consecutively, can serve as a starting scheme of the theoretical description in each case [1-4].

From microscopic point of view, however, a displacive type or order/disorder type PT are considered to be two opposite limiting mathematical cases for the energetic system, which is never realized in real systems. Real materials show intermediate behavior, and their pertaining to one of the two limiting cases can be used as "zero" approximation. The deviation from both of these limiting schemes is caused by 
the peculiarities of the microscopic energy of a specific system. It is desirable to find a certain "key" to the decoding, at least approximate, of the relation between microscopic parameters of a real system with the character of the observed PT in the system investigated. Herein we can systematize a nonuniversal behavior of crystals' properties at the phase $\mathrm{PT}$, i. e. their behavior off the critical region - in the most convenient (for majority cases) temperature interval for experimental investigations.

Experimental data for the family of $\mathrm{Sn}_{2} \mathrm{P}_{2} \mathrm{~S}(\mathrm{Se})_{6}$ crystals of uniaxial ferroelectrics motivate the study of this system using different theoretical approaches. In a composition - temperature phase diagram of solid solutions $\mathrm{Sn}_{2} \mathrm{P}_{2}\left(\mathrm{Se}_{x} \mathrm{~S}_{1-x}\right)_{6}$ of these crystals, the Lifshitz point is present $[5,6]$ at which the paraelectric, ferroelectric and incommensurate (IC) phases meet. There is also a virtual tricritical point which together with long range dipole interaction determines a specific critical behavior of thermodynamic properties in the region of phase transitions $[7,8]$. Furthermore, this family displays the crossover effects from displacive to order/disorder type of phase transition $[9,10]$. The investigations of $\mathrm{Sn}_{2} \mathrm{P}_{2} \mathrm{~S}_{6}$ ceramics [11] have shown a decrease of the PT temperature at lowering of the dimensions of microcrystals.

To compare different models used for the discussed ferroelectrics analysis is the aim of this paper. Thereby, arguments were found for performing calculations by Monte Carlo (MC) in a rather simple discrete Ising-like model of dimension dependence for phase transitions.

\section{Thermodynamic and dynamic properties of $\mathrm{Sn}_{2} \mathrm{P}_{2} \mathrm{~S}(\mathrm{Se})_{6}$ fer- roelectrics: illustration of polycritical points and displacive- order/disorder crossover coincidence on the phase diagram}

From the set of experimental data it follows that in the $\mathrm{Sn}_{2} \mathrm{P}_{2} \mathrm{~S}_{6}$ and $\mathrm{Sn}_{2} \mathrm{P}_{2} \mathrm{Se}_{6}$ ferroelectrics, the phase transitions are placed in the region of crossover from displacive type to order/disorder type. Herein, by substitution of sulfur by selenium, the PT character is approached to order/disorder type. Let's see the experimental data and general estimations based on them.

On the temperature-concentration diagram for $\mathrm{Sn}_{2} \mathrm{P}_{2}\left(\mathrm{Se}_{x} \mathrm{~S}_{1-x}\right)_{6}$ solid solutions the Lifshitz point is reached (figure 1) - ferroelectric PT of the second order (for $\mathrm{Sn}_{2} \mathrm{P}_{2} \mathrm{~S}_{6} T_{0} \sim 337 \mathrm{~K}$ ) at $x>0.28$ splits into the second and into the first order transitions, that limit the incommensurate (IC) phase (for

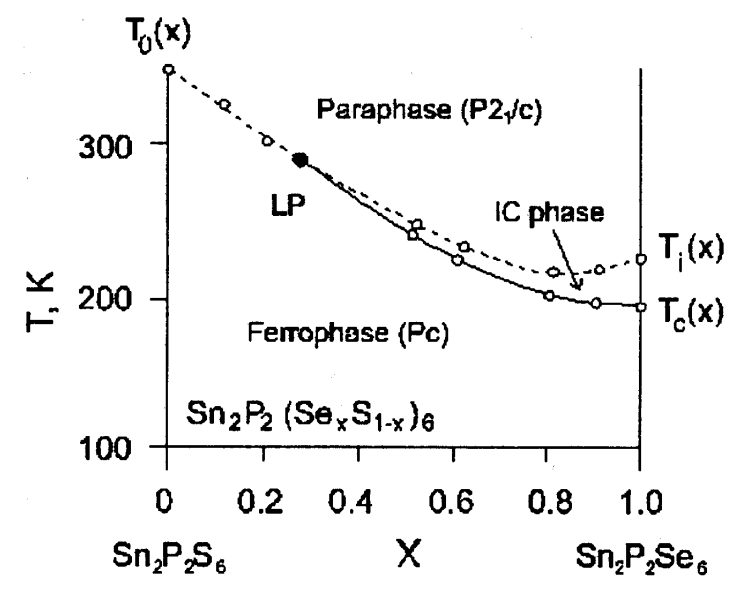

Figure 1. Phase diagram of the solid solutions $\mathrm{Sn}_{2} \mathrm{P}_{2}\left(\mathrm{Se}_{x} \mathrm{~S}_{1-x}\right)_{6}$. LP - Lifshitz point; dashed line - second order phase transitions; continuous line - first order phase transitions $[5,6]$. 


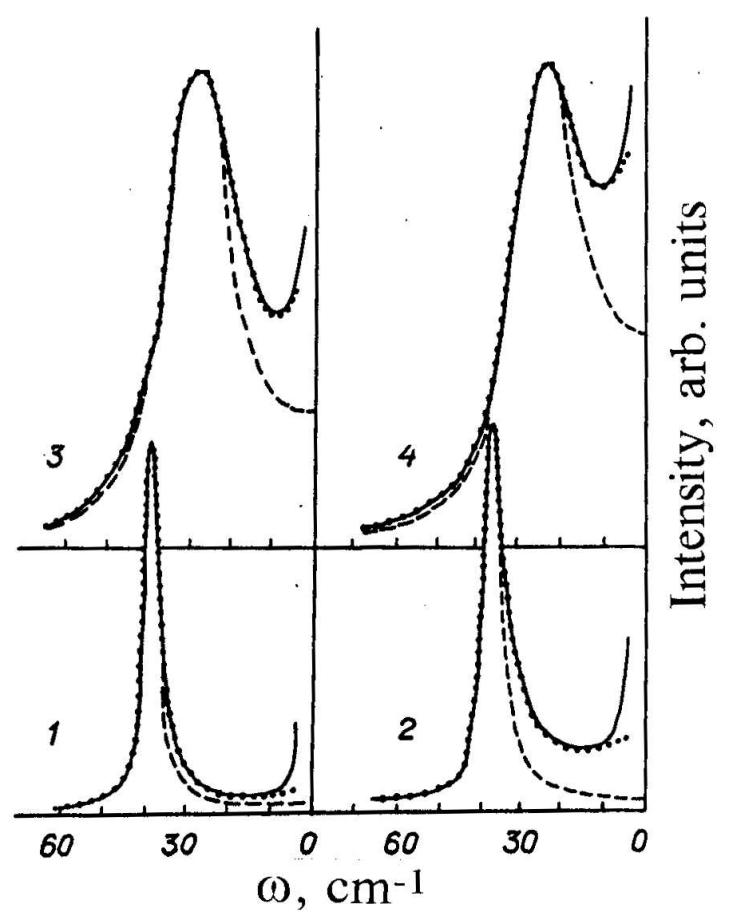

Figure 2. Soft mode spectral line in the ferroelectric phase of $\mathrm{Sn}_{2} \mathrm{P}_{2} \mathrm{~S}_{6}$ at: $1-80 \mathrm{~K}$; $2-233 \mathrm{~K} ; 3-293 \mathrm{~K} ; 4-313 \mathrm{~K}$. Solid line - experiment, dashed line - Lorentz contour fitting, points - fitting by a response function with account of an interaction between oscillator and relaxator [6].

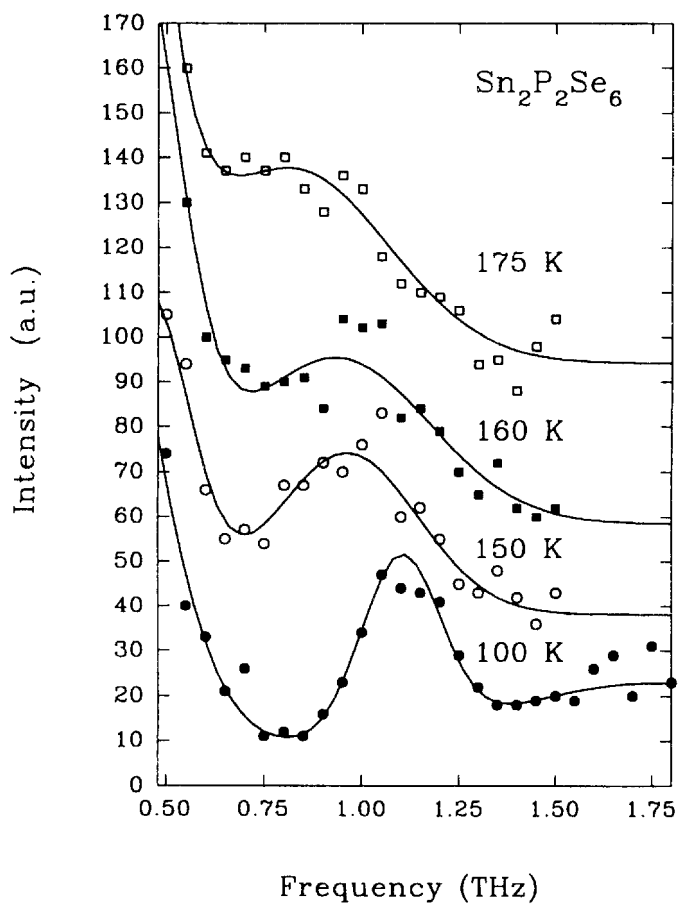

Figure 3. Soft mode behavior for the ferroelectric phase of $\mathrm{Sn}_{2} \mathrm{P}_{2} \mathrm{Se}_{6}$ from a neutron scattering data at the (3 0 1) Brillouin zone center. The full lines are damped harmonic oscillator fits [13].

$\left.\mathrm{Sn}_{2} \mathrm{P}_{2} \mathrm{Se}_{6} T_{i} \sim 221 \mathrm{~K}, T_{c} \sim 193 \mathrm{~K}\right)$. The modulation period of spontaneous polarization in the IC phase equals $12-15$ primitive cells $[5,6]$.

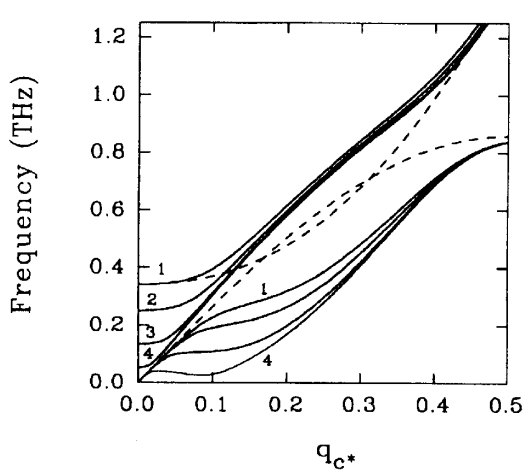

Figure 4. The model simulation of the incommensurate instability on the neutron scattering data for $\mathrm{Sn}_{2} \mathrm{P}_{2} \mathrm{Se}_{6} .1-$ $235,2-205,3-180,4-171 \mathrm{~K}[13]$.
For $\mathrm{Sn}_{2} \mathrm{P}_{2} \mathrm{~S}_{6}$ and $\mathrm{Sn}_{2} \mathrm{P}_{2} \mathrm{Se}_{6}$ in the Raman scattering spectra (figure 2) [6], and in the neutron scattering spectra (figures 3 and 4) [12,13], the soft optical mode is observed. According to the temperature dependence of a dielectric susceptibility [8] in the paraelectric phase of these compounds (figure 5) the Curie-Weiss constant is in the range of $C=0.5-0.7 \cdot 10^{5} \mathrm{~K}$. Such characteristics are peculiar for the displacive type PT. At the same time, for $\mathrm{Sn}_{2} \mathrm{P}_{2} \mathrm{~S}_{6}$ in the Brillouin scattering [6] and in neutron scattering [12] spectra, a central peak is observed which testifies to the presence 


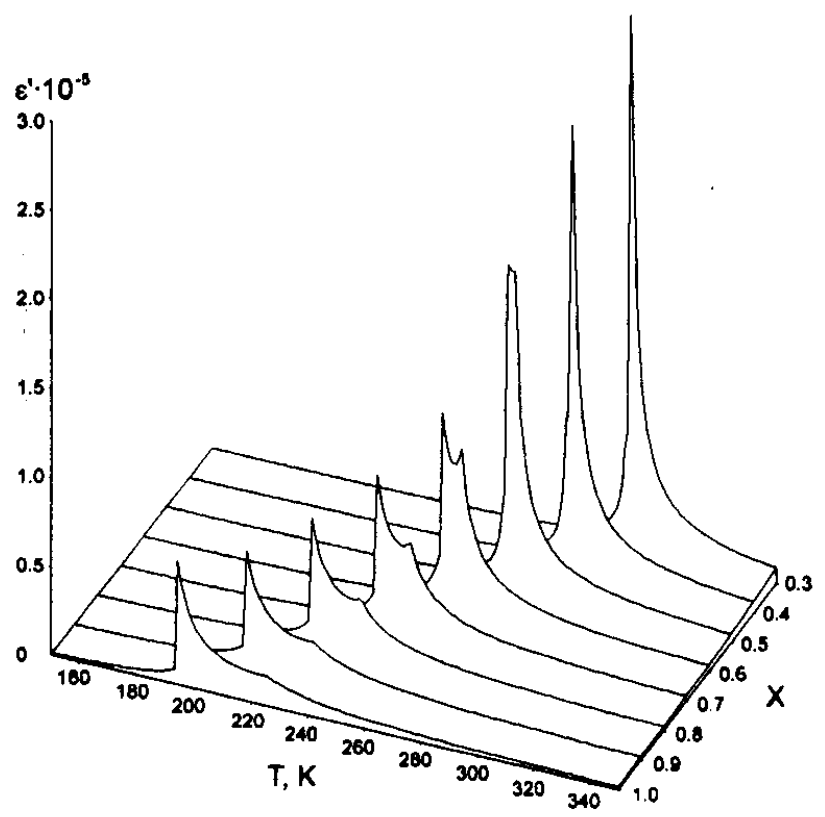

a

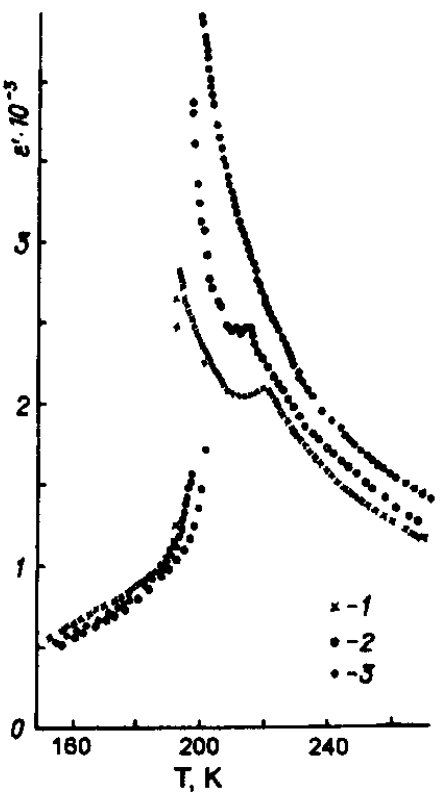

b

Figure 5. The calculated concentrational evolution of dielectric constant temperature dependence for the crystals $\mathrm{Sn}_{2} \mathrm{P}_{2}\left(\mathrm{Se}_{x} \mathrm{~S}_{1-x}\right)_{6}: 1-x=1,2-0.9,3-0.8$ [8] (a) in the mean field approximation and (b) determined experimentally.

of a relaxation component of critical dynamics. This component is also observed in a spectral response of the soft phonons both for $\mathrm{Sn}_{2} \mathrm{P}_{2} \mathrm{~S}_{6}$ and $\mathrm{Sn}_{2} \mathrm{P}_{2} \mathrm{Se}_{6}$ (figures 2 and 3). Calorimetric studies [14] of both crystals reveal a large entropy of the phase transitions: $8.6 \mathrm{~K}^{-1} \mathrm{~mol}^{-1}$ for the ferroelectric-paraelectric transition in $\mathrm{Sn}_{2} \mathrm{P}_{2} \mathrm{~S}_{6}$, and 8.5 and $1.5 \mathrm{~K}^{-1} \mathrm{~mol}^{-1}$ for the ferroelectric-IC transition and IC phase-paraelectric phase transitions, correspondingly, in case of $\mathrm{Sn}_{2} \mathrm{P}_{2} \mathrm{Se}_{6}$ (figure 6). These entropy values are close to $2 R \ln 2=11.4 \mathrm{~K}^{-1} \mathrm{~mol}^{-1}$ which corresponds to a positional disordering of $\mathrm{Sn}^{2+}$ cations.
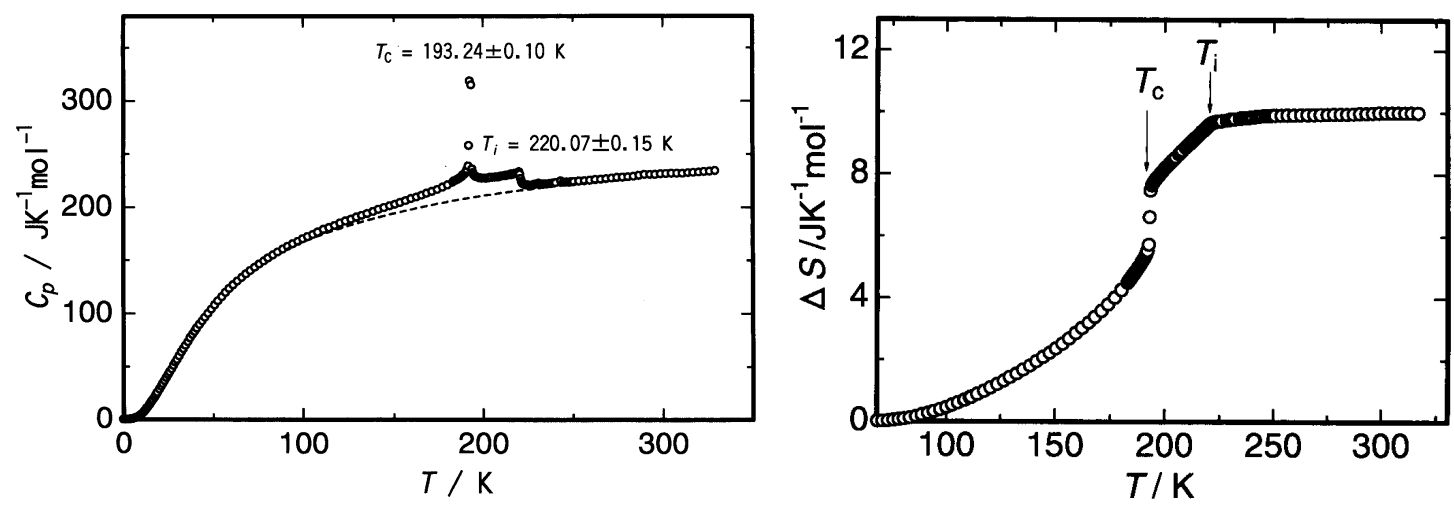

Figure 6. The heat capacity and the transition entropy for $\mathrm{Sn}_{2} \mathrm{P}_{2} \mathrm{Se}_{6}$ as a function of temperature [14]. 


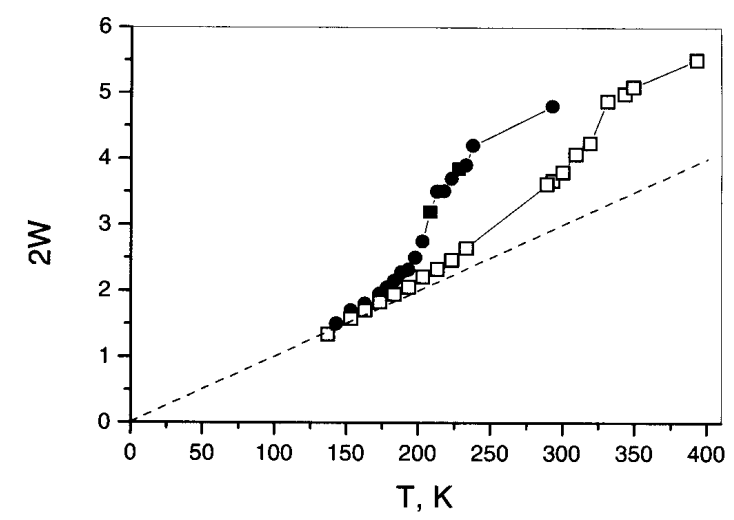

Figure 7. The temperature dependencies of the Debye-Waller factor for $\mathrm{Sn}_{2} \mathrm{P}_{2} \mathrm{Se}_{6}$ (full circles) and $\mathrm{Sn}_{2} \mathrm{P}_{2} \mathrm{~S}_{6}$ (open squares) from the ${ }^{119} \mathrm{Sn}$ Mossbauer spectroscopy [15].

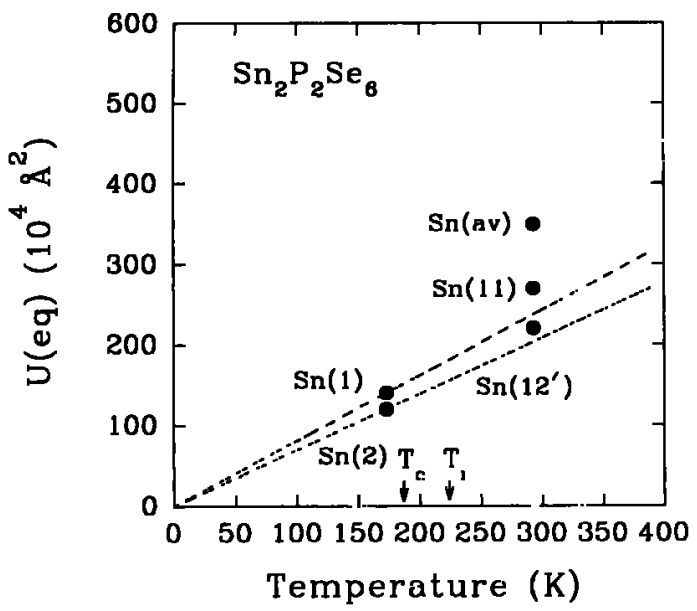

According to the investigations [15] of Mossbauer spectra of ${ }^{119} \mathrm{Sn}$ isotope, the temperature dependence of a meansquare amplitude of heat shifts of ferroactive Sn atoms under the heating in the ferroelectric phase deviates from a linear, and in paraelectric phase it reaches $0.34 \AA$ (figure 7 ). These data confirm the speculation about the possible existence of a two-well potential for Sn atoms in the paraelectric phase of the crystals studied. The X-ray structural studies also confirm a substantial increase of the mean-square shifts for Sn atoms at the transition into paraelectric phase, both in $\mathrm{Sn}_{2} \mathrm{P}_{2} \mathrm{~S}_{6}$ [16] and $\mathrm{Sn}_{2} \mathrm{P}_{2} \mathrm{Se}_{6}[17,18]$ (figure 8).

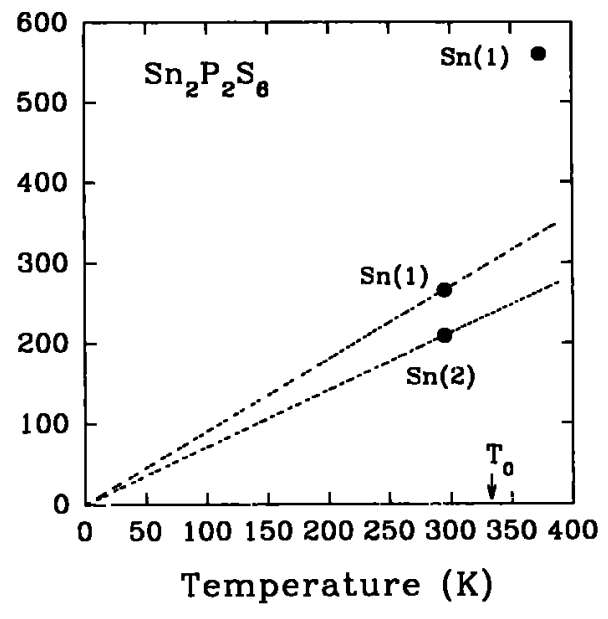

Figure 8. The temperature dependencies of the parameters of isotropic atomic heat displacements for $\mathrm{Sn}^{2+}$ cations in $\mathrm{Sn}_{2} \mathrm{P}_{2} \mathrm{Se}_{6}$ and $\mathrm{Sn}_{2} \mathrm{P}_{2} \mathrm{~S}_{6}$ crystals on X-ray data [17].

The comparison of the structures of paraelectric and ferroelectric phases for $\mathrm{Sn}_{2} \mathrm{P}_{2} \mathrm{Se}_{6}$ crystals was made in [17] and [18] by means of a detailed analysis of X-ray data. According to [18] the change of the crystal structure at ferroelectricparaelectric transition is due to the shift of the Sn atoms to centrosymmetric positions. Heat vibrations of Sn atoms in the paraelectric phase are very anharmonic. However, the attempt made in [18] to describe the structure of paraelectric phase based on the assumption of the presence of Sn atoms disordering in two positions, failed to improve the quality of comparison of the X-ray diffraction data. In the mean time, the X-ray diffraction data obtained in [17] had been analyzed more precisely in the model assuming Sn atoms disordering in two positions, separated by 
$0.34 \AA$, and the shift of the average distance between these positions separated by $0.31 \AA$ and by $0.28 \AA$, correspondingly, for two Sn pairs relative to the positions of Sn atoms in the ferroelectric phase at $173 \mathrm{~K}$. Thus, direct data on the structure of $\mathrm{Sn}_{2} \mathrm{P}_{2} \mathrm{~S}_{6}$ and $\mathrm{Sn}_{2} \mathrm{P}_{2} \mathrm{Se}_{6}$ crystals testify to a substantially anharmonic potential for $\mathrm{Sn}^{2+}$ cations in paraelectric phase. However, certain proofs of a two-well potential have been obtained only for $\mathrm{Sn}_{2} \mathrm{P}_{2} \mathrm{Se}_{6}$.

While analysing the experimental data, it is convenient to start with the simplest approximations. Such approximations serve as a background of the so-called $\Phi^{4}$ model [1-3]. This model can be generalized by including a many-component order parameter, taking into account the interactions with different radii, and also, if it is needed, long-range dipole-dipole interactions, by accounting for the interaction with elastic deformations. The temperature behavior of $\Phi^{4}$ model is determined by $E_{0}$ and $J$ parameters, that measure the depth of the potential well and the strength of interaction between the neighbors. The behavior of the system is determined by relation $\varepsilon=J / E_{0}$ : at $\varepsilon \ll 1$, the system approaches the order/disorder regime and its behavior is adequate to the behavior of a lattice of local spins. At the opposite border of large values of $\varepsilon$, the picture of the soft phonons becomes adequate and the approximation of self-consistent phonons becomes exact at $\varepsilon \rightarrow \infty$. In the mean field approach, the crossover border was determined $\varepsilon=1 / 3[3]$.

For the estimation of the PT type in specific crystals it is convenient to use the empirical factor proposed in [3]:

$$
R=\left(2 \Delta S / k_{\mathrm{B}}\right)^{-0.5}
$$

which is determined by entropy and Boltsmann constant. The relation between the factor $R$ and the characteristic $\varepsilon$ (figure 9) was determined by numerical calculation.

In the framework of the simplest one-ion model [9] while classifying the PT character in specific objects it is also convenient to determine the relation between the heat energy $k T_{0}$ (at temperature of phase transition $T_{0}$ ) and the barrier energy $U_{m}$ for a two-well potential of a ferroactive particle. The change of the energy $U_{f}$ of interaction of the neighboring elementary cells, which appears at the flipping of the ferroactive cation across the potential barrier within one of these cells, is an important characteristic. For PT of displacive type, $k T_{0}>U_{m}$ and $U_{f}>U_{m}$. For order/disorder type $\mathrm{PT}$, the opposite relations are expected: $k T_{0}<U_{m}$ and $U_{f}<U_{m}$.

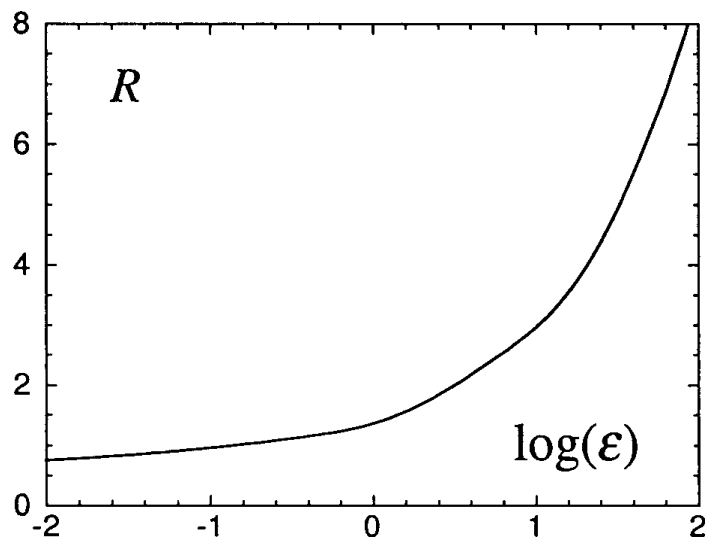

Figure 9. Temperature dependence of the empiric factor $R$ (was determined by the relation (1)) in $\Phi^{4}$ model vs. the displacive degree $\varepsilon[3]$.

As follows from the estimations performed in [9], that are based on the thermodynamic and spectroscopic data, for $\mathrm{Sn}_{2} \mathrm{P}_{2} \mathrm{~S}_{6}$ crystal, the energy of the barrier for 
the normal coordinate of the soft phonon $U_{m}=4 \cdot 10^{-21} \mathrm{~J}$, which is close to $k T_{0}=$ $6 \cdot 10^{-21} \mathrm{~J}$ at $T_{0}=337 \mathrm{~K}$. Thus, the ferroelectric crystal $\mathrm{Sn}_{2} \mathrm{P}_{2} \mathrm{~S}_{6}$ has the phase transition in the region of crossover from displacive type to order/disorder type.

Available experimental data are not sufficient to perform similar estimations for $\mathrm{Sn}_{2} \mathrm{P}_{2} \mathrm{Se}_{6}$ crystal. Not mentioning the possibility of using the relation (1) to estimate the PT character in $\mathrm{Sn}_{2} \mathrm{P}_{2} \mathrm{~S}_{6}$ and $\mathrm{Sn}_{2} \mathrm{P}_{2} \mathrm{Se}_{6}$ crystals. According to the calorimetric data [14] for these crystals, the entropy of the transition from paraelectric phase to ferroelectric phase equals 8.6 and $10 \mathrm{~J} \mathrm{~K}^{-1} \mathrm{~mol}^{-1}$. Herein, the empiric factor $R=0.69$ for $\mathrm{Sn}_{2} \mathrm{P}_{2} \mathrm{~S}_{6}$ and $R=0.64$ for $\mathrm{Sn}_{2} \mathrm{P}_{2} \mathrm{Se}_{6}$. From the dependence $R$ vs. $\log \varepsilon$ (figure 9 ) we can find that $\varepsilon \leqslant 1 / 3$ for sulfide and selenide compounds. Remember that in the mean-field approach, the border of crossover from displacive type of PT to order/disorder type was determined $\varepsilon \sim 1 / 3[3]$.

For $\mathrm{Sn}_{2} \mathrm{P}_{2} \mathrm{Se}_{6}$, compound the presence of the intermediate incommensurate phase itself is probably more important for the actual soft mode vs. order/disorder behavior. To consider this situation, a detailed analysis of the order/disorder vs. soft mode crossover in the DIFFOUR (Discrete Frustrated $\Phi^{4}$ ) model was performed in papers $[19,20]$.

The crossover situation for $\mathrm{Sn}_{2} \mathrm{P}_{2} \mathrm{~S}(\mathrm{Se})_{6}$ ferroelectrics actually implies that these compounds should reveal a soft mode, which, however, does not soften completely, which is really observed in the experiment (figures 2 and 3) $[6,12,13]$. In this regime, the ratio $r$ of slopes of the temperature dependencies of the soft mode frequency could be high enough, depending on the actual form of the phonon dispersion. This agrees with scattering data value $r \sim-7[12,13]$ experimentally estimated from inelastic neutron, and is also responsible for the large value of the ratio of Curie-Weiss constants, that is observed experimentally (figure 5) [8]. It can be also claimed that the quartic coefficient of the Landau potential for ferroelectric $\mathrm{Sn}_{2} \mathrm{P}_{2} \mathrm{~S}_{6}$ should be considered as temperature dependent on the temperature scales comparable with $T_{0}$. The reduction of its value at $T_{0}$ should be significant, so that the sixth-order term in the Landau expansion may really play an important role in the vicinity of $T_{0}$ [3]. This suggests that the crossover behavior could be responsible for a nonstandard temperature dependence of the spontaneous polarization. Their saturation is achieved faster than it would be expected for a usual second order displacive phase transition. According to the simulations [9], an effective value of index $\beta$ in a dependence $P_{\mathrm{S}} \sim\left(T_{0}-T\right)^{\beta}$ should be smaller than 0.5.

Hence, based on the review of the dynamic and thermodynamic properties of $\mathrm{Sn}_{2} \mathrm{P}_{2} \mathrm{~S}_{6}$ family ferroelectrics and taking into account their analysis in the framework of simple models we can focus our attention on the presence of the following interesting correlations. Approaching the Lifshitz point on the state diagram of the solid solutions $\mathrm{Sn}_{2} \mathrm{P}_{2}\left(\mathrm{Se}_{x} \mathrm{~S}_{1-x}\right)_{6}$ by increasing $x$ to $x_{\mathrm{LP}}=0.28$ determines the increase of the temperature interval near the phase transition temperature where the effect of strongly developed fluctuations of the order parameter is important. This determines the deviation of the thermodynamic properties temperature behavior from the one expected in the mean field approaches. Such deviation, particularly, appears in the decreasing of the effective value of critical index $\beta$ for temperature de- 
pendence of the order parameter. At the rise of selenium concentration, the PT from paraelectric phase to ferroelectric phase approaches the tricritical point at $x=0.6$ $[7,8]$. This also assists in a considerable decrease of the named index $\beta$.

At the same time, it is known that at approaching the LP, the dispersion of the rigidity for the order parameter fluctuations in the direction of modulation decreases, i.e., the intercell interactions decrease. On the other hand, the coefficient at the invariant of the fourth power in the thermodynamic potential decreases at approaching the tricritical point. Thereby, obviously, the coefficient at the addend of the same power in the configuration energy function also decreases i.e., the appearance of local anharmonism changes. The latter fact determines the increase of the potential barrier value $E_{0}$ for the local two-well potential of the ferroactive particle. Thus, at approaching LP and TCP on the state diagram of the solid solutions $\mathrm{Sn}_{2} \mathrm{P}_{2}\left(\mathrm{Se}_{x} \mathrm{~S}_{1-x}\right)_{6}$, a simultaneous intercell interaction decreases and the height of potential barrier in the local potential increases, which determines the change of the phase transition character from displacive type to order-disorder type. Such a crossover is connected with the deviation from simple $\Phi^{4}$ model. The deviation testify, as was shown by numerical analysis [3], that at a successive consideration of the thermodynamic potential it is needed to take into account the nonlinearity in the temperature dependence of the coefficient at the quadratic invariant, as well as the presence of the temperature dependence for the coefficient at the quartic invariant. Accounting for these dependencies also explains the decrease of the critical index for the temperature dependence of the order parameter at the change of the second order phase transition character in the direction to order/disorder type.

\section{Simulations based on the microscopic models}

Models based on the discrete structure of the lattice can provide a way to achieve a physical understanding of the processes on the atomic level of the crystal, which goes beyond a mere phenomenological description. Such microscopic models can be handled analytically or numerically. The models, which have been closely studied with respect to the application to experimentally investigated substances, are discussed in [4]. Each model variable - either continuous as in the case of the DIFFOUR models $[4,19,20]$ or discrete as in the case of pseudo spin models [4] - usually describes one degree of freedom per lattice site.

The experimental phase diagrams, however, are spanned by external quantities like temperature, concentration, pressure or applied fields. Thus, it is necessary to find a mapping between the experimental quantities and the theoretical parameters in order to compare the experimental and the theoretical phase diagrams. In what follows we will present a procedure for transforming a two-dimensional theoretical phase diagram from model parameters to temperature-concentration (pressure) variables.

A generalization of the simplest possible discrete DIFFOUR model with the Lifshitz point on the phase diagram was made in papers $[19,20]$ with the aim to explain the experimental data for $\mathrm{Sn}_{2} \mathrm{P}_{2}\left(\mathrm{Se}_{x} \mathrm{~S}_{1-x}\right)_{6}$ crystals. Herein the phase dia- 
gram for $0 \leqslant x \leqslant 1$ and the temperature dependence of the modulation wave vector for $\mathrm{Sn}_{2} \mathrm{P}_{2} \mathrm{Se}_{6}$ were calculated. In order to satisfactorily reproduce the experimental data, a nonlinear coupling with nearest neighbors is taken into account [20]. The incommensurability in this model arises due to the frustration between the nearest neighbor and the next-nearest neighbor interactions. However, the tricriticality is not taken into account in the generalized DIFFOUR model $[19,20]$. Of course, the inclusion of a sixth order term to the potential energy that is needed to describe the herein important tricritical fluctuations, determines no linear mapping of the calculated phase diagram on the experimental $T-x$ diagram. Thereby, more experimental data, in particular for the modulation wave vector in the interval $0.28 \leqslant x \leqslant 1$, are needed for this mapping.

In papers $[10,21]$ to explain the specificity of the phase diagrams of $\mathrm{Sn}_{2} \mathrm{P}_{2} \mathrm{~S}(\mathrm{Se})_{6}$ ferroelectrics there was proposed a dipole Ising model of the PT mechanisms using the calculations according to the standard procedure of Monte Carlo [22]. This model was developed on the basis of structural data of these crystals in the paraelectic phase. The basis of the model is as follows: a dipole, which is parallel to the crystalographic axis a and is located in $\mathrm{Sn}^{2+}$ position in the paraelectic phase, corresponds to each of the four atoms of ferroactive $\mathrm{Sn}^{2+}$ sublattice. To consider the dipole long range interaction around the dipole, an area of simple form is singled out. Within this area the interaction between the dipoles is calculated precisely. The interaction with the other dipoles is considered by introducing a local field. The macroscopic sample was taken as a limited Ising lattice with the imposed periodical boundary conditions (Monte Carlo cell). For a better coincidence with the experiment, an additional parameter was introduced to the basic model, i.e., correction for the short range interaction that encapsulates other (except the dipole) short range forces. The mentioned correction could be considered as a degree of deviation of a real object from the dipole Ising model that is adequate to the case of an ideal order/disorder PT. Using the calculations made by MC techniques it has been found that when certain values of short range interaction coefficient for $\mathrm{Sn}_{2} \mathrm{P}_{2} \mathrm{~S}_{6}$ and $\mathrm{Sn}_{2} \mathrm{P}_{2} \mathrm{Se}_{6}$ are selected (the correction is lower for the latter compound, which shows a more pronounced order/disorder character of the PT), the temperatures of PT's are as follows: for $\mathrm{Sn}_{2} \mathrm{P}_{2} \mathrm{~S}_{6}$ model $T_{0} \sim 10.3$; for $\mathrm{Sn}_{2} \mathrm{P}_{2} \mathrm{Se}_{6}$ model $T_{c} \sim 8.7, T_{i} \sim 9.7$ (at the effective temperatures). These data are in satisfactory agreement with the results of the experimental diagram (figure 1). From the dipole model point of view, the peculiarities of this diagram - the existence of incommensurate phase in $\mathrm{Sn}_{2} \mathrm{P}_{2} \mathrm{Se}_{6}$ and its absence in $\mathrm{Sn}_{2} \mathrm{P}_{2} \mathrm{~S}_{6}$ crystal - are based on the difference of geometric locations of the ferroactive sublattice atoms.

We should note that the application of the dipole model of the Ising type provides the PT modelling of the order/disorder type, whereas the real crystals demonstrate a somewhat intermediate character of PT - between order/disorder and displacive type. Furthermore, it was assumed that a statistical approach to the evaluation of thermodynamic functions and a phase diagram of the system permits to disregard the peculiarities of the dynamic motion of separate atoms.

Mean-field models were also used for the analysis of the properties of 
$\mathrm{Sn}_{2} \mathrm{P}_{2}\left(\mathrm{Se}_{x} \mathrm{~S}_{1-x}\right)_{6}$ ferroelectrics $[7,8,23]$. Taking into account the interactions between spontaneous polarization and elastic deformations, as well as taking into account the higher harmonics of the modulation of the polarization in the IC phase, the behavior of the thermodynamic functions (spontaneous polarization, dielectric susceptibility, heat capacity) along the phase diagram was described $[7,8]$. It was shown $[23]$ that the temperature dependence of the wave vector modulation can be correctly described simultaneously with the anomalous heat capacity only when the nonlinear properties of the order parameter distribution are taken into account.

For a type II incommensurate phase, that was determined by symmetry for proper $\mathrm{Sn}_{2} \mathrm{P}_{2}\left(\mathrm{Se}_{x} \mathrm{~S}_{1-x}\right)_{6}$ ferroelectrics, the PT instability appears to be off (but still near) the Brillouin zone center. A coupling of transverse optic (TO) and transverse acoustic (TA) phonon branches lies at the origin of this IC instability, that was clearly demonstrated by neutron scattering data (figure 4) [13]. We wonder which is more important for the mixed displacive-order/disorder type of the PT in the crystals considered, a dipolar coupling among the displacements of $\mathrm{Sn}^{2+}$ cations, or the TO-TA coupling? This question was considered in the paper [24] within a model, which permits to comment on the physical relevance of both these approaches to $\mathrm{Sn}_{2} \mathrm{P}_{2} \mathrm{~S}_{6}$. It was concluded, that the origin of the IC instability and the value of the wave vector modulation at $T_{i}$ is indeed uniquely determined by the dipolar interactions between $\mathrm{Sn}^{2+}$ cations. Anions $\left[\mathrm{P}_{2} \mathrm{~S}(\mathrm{Se})_{6}\right]^{4-}$ have to be taken into account only if the low frequency phonon dispersion (TO-TA coupling) is also to be described.

So, we could again conclude that the simplest dipole Ising-like models could be used for the simulation of the phase diagram and of some thermodynamic properties of $\mathrm{Sn}_{2} \mathrm{P}_{2} \mathrm{~S}_{6}$ type ferroelectrics. Further on we will present the ANNNI adapted model for the simulation of the PT temperature dependence as the function of the dimension of microcrystals. Such an analysis is quite interesting because according to experimental data [11] on the dielectric properties of $\mathrm{Sn}_{2} \mathrm{P}_{2} \mathrm{~S}_{6}$ ceramics at the average dimensions of microcrystals about $1 \mu \mathrm{m}$, the ferroelectric PT temperature is already about $325 \mathrm{~K}$, instead of $T_{0} \sim 337 \mathrm{~K}$ for the bulk samples of $\mathrm{Sn}_{2} \mathrm{P}_{2} \mathrm{~S}_{6}$.

The described numerical experiments were executed over the dipole model of $\mathrm{Sn}_{2} \mathrm{P}_{2} \mathrm{~S}(\mathrm{Se})_{6}$ crystals with four pseudo-dipoles in the primitive cell $[10,21,25]$. The long range character of dipole interaction was taken into account under the classic scheme [25]. The calculations conducted have shown a good consent with the phase diagram obtained experimentally for a case, when the sample of macroscopic dimensions was considered. An essential drawback of this model is the failure at attempting to simulate microscopic samples. At the removal of a Lorentz local field by a ground state of the model we observe a modulated phase.

To study the PT's in microcrystals, an ANNNI (Axial Next Nearest Neighbors Ising) model is widely used $[4,26]$. In the framework of this model there were demonstrated such effects as the relation of temperature of bulk PT between ferroelectrics and paraelectric phases to the dimensions of the model lattices, the influence of a surface of a microscopic sample on phase changes. The basic sense of returning to ANNNI model in the given paper is binding the known abstract model to the actual phase diagram of microcrystals such as Sn2P2S6. 


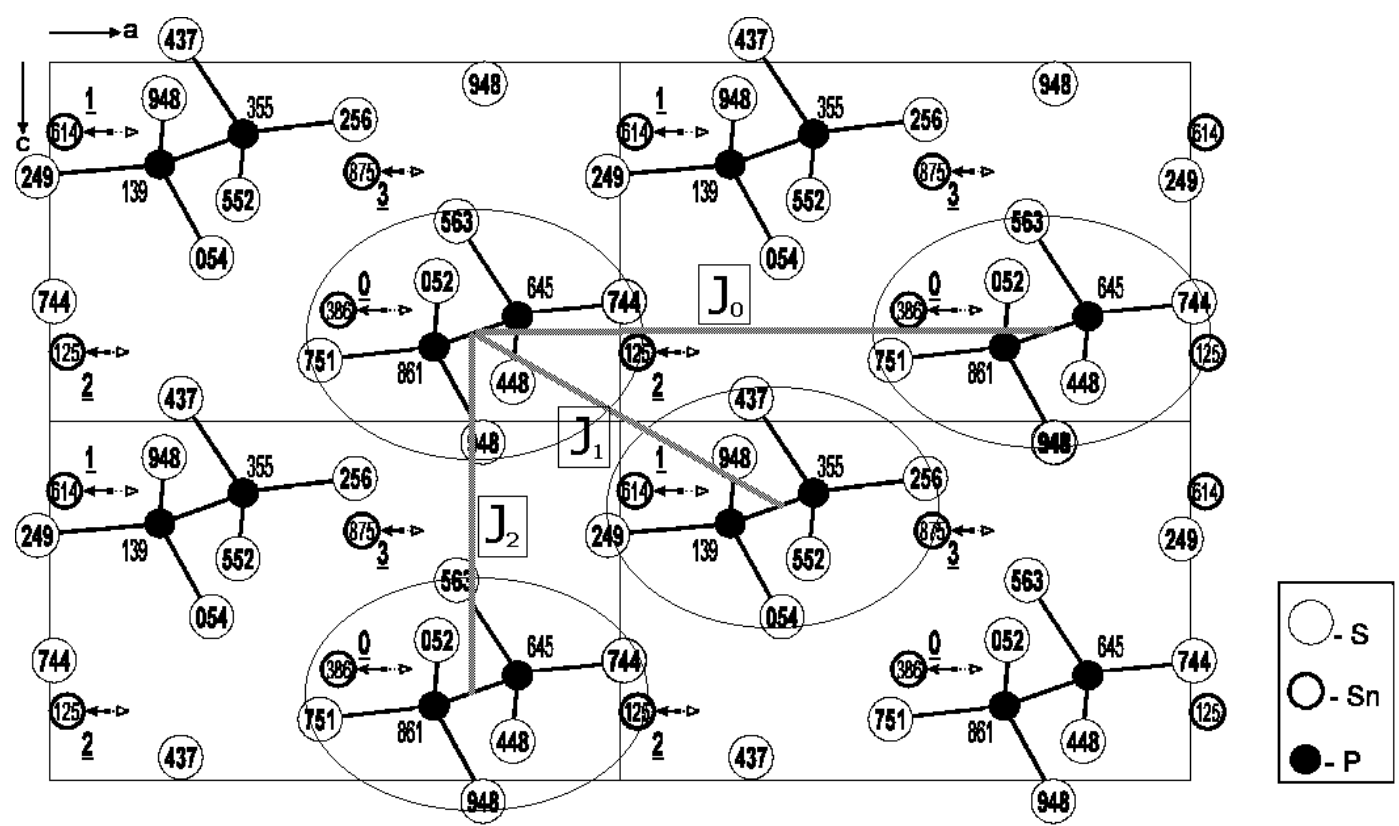

Figure 10. The projection of the crystal structure of $\mathrm{Sn}_{2} \mathrm{P}_{2} \mathrm{~S}_{6}$ in the ferroelectric phase on the plane (010). The altitude of atoms is indicated in thousand shares of a period. The arrows mark the atoms of the ferroelectric sublattice.

To adjust the ANNNI model in order to make it distinct from the cubic lattice of dipoles, the scheme of interactions was changed. Hereinafter we shall call such a model the ANNNI-adapted. We can obtain the coefficients of ANNNI-adapted model by involving periodic boundary conditions and by comparing the resulting phase diagram with the experimental phase diagram of $\mathrm{Sn}_{2} \mathrm{P}_{2} \mathrm{~S}_{6}$ (figure 1). With the coefficients obtained, we investigate the phase changes in microcrystal models with the different dimension of a Monte Carlo cell and constant boundary conditions.

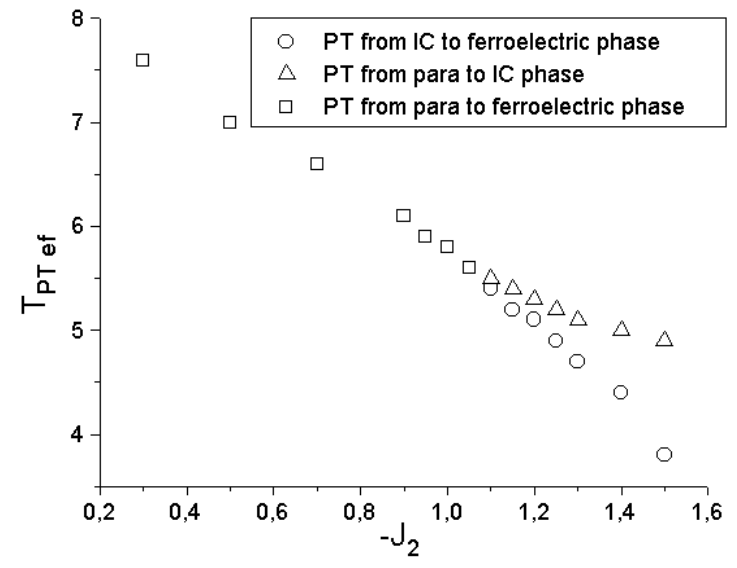

Figure 11. The phase diagram of ANNNI-adapted model in the area of a Lifshitz point at $J_{0}=J_{1}=1$.

We shall link an effective dipole to the molecular group of atoms $\mathrm{Sn}_{2} \mathrm{P}_{2} \mathrm{~S}_{6}$ (four such groups are marked in figure 10 by ellipses), its electrical dipole moment being directed along the axis a. In the spirit of concurrent interactions in the framework of ANNNI model we define the interactions in our model. They are represented by coefficients: $J_{0}>0$ - between dipoles located on an axis $\mathbf{a}$, $J_{1}>0$ - between the nearest adjacent dipoles, $J_{2}<0$ - between the nearest dipoles with negative interaction. For a simplicity, let's assume $J_{0}=J_{1}=1$.

Unlike in the earlier papers [4] where the phase diagram of ANNNI model on 
a cubic lattice was obtained using the method of a mean field, we have done a more correct binding of the coefficients of the adapted ANNNI model to the crystal lattice of $\mathrm{Sn}_{2} \mathrm{P}_{2} \mathrm{~S}_{6}$ compound. We have constructed its precise phase diagram in the area of a Lifshitz point by a Monte Carlo method (figure 11).

It is necessary to focus on the boundary conditions used while performing the calculations: to simulate the bulk crystal we used periodic boundary conditions (PBC), while to simulate microcrystals, free boundary conditions were used.

The coefficients for $\mathrm{Sn}_{2} \mathrm{P}_{2} \mathrm{~S}_{6}$ model could be evaluated by comparing the experimental diagram of the solid solutions $\mathrm{Sn}_{2} \mathrm{P}_{2}\left(\mathrm{Se}_{x} \mathrm{~S}_{1-x}\right)_{6}$ (figure 1) with the calculations depicted in figure 11, and using the proportional relation between $J_{2}$ and $x$. For $x=1$ ( $\mathrm{Sn}_{2} \mathrm{P}_{2} \mathrm{Se}_{6}$ compound), the width of the IC phase over temperature is $\left(T_{i}-T_{c}\right) / T_{i} \sim 0.13$ and $x=0.28$ (Lifshitz point), the IC phase disappears. Herein we can define $J_{2}=-0.92$ for $x=0$.

With the parameters obtained we have performed a series of numerical tests under the ANNNI-adapted model for different dimensions of the Monte Carlo cell. The results are depicted in figure 12. We can see the increase of the calculated effective temperature $T_{\text {ef }}$ of PT from ferroelectric to paraelectric phase with the growth of this value on saturation, defined by the models with $\mathrm{PBC}$, i.e. the models of a bulk sample of $\mathrm{Sn}_{2} \mathrm{P}_{2} \mathrm{~S}_{6}$. These results are in the qualitative consent with experimental data [11].

ANNNI-adapted model has the main severe drawback, i.e., a weak affection to an actual lattice of such a complex crystal as $\mathrm{Sn}_{2} \mathrm{P}_{2} \mathrm{~S}_{6}$. To overcome this drawback the following short range model for $\mathrm{Sn}_{2} \mathrm{P}_{2} \mathrm{~S}_{6}$ was offered: the pseudo-dipoles are arranged just as in the latter case, the scheme of interactions as well as in ANNNI model is constructed on the nearest and on the next nearest neighbors, but the coefficients of interactions $J_{1}<0, J_{2}<0, J_{3}>0, J_{4}>0$ are calculated under the formula of dipole-dipole interaction on the basis of the geometrical data of the crystal lattice (figure 13). Moreover, the positive correction $\Delta J$ on

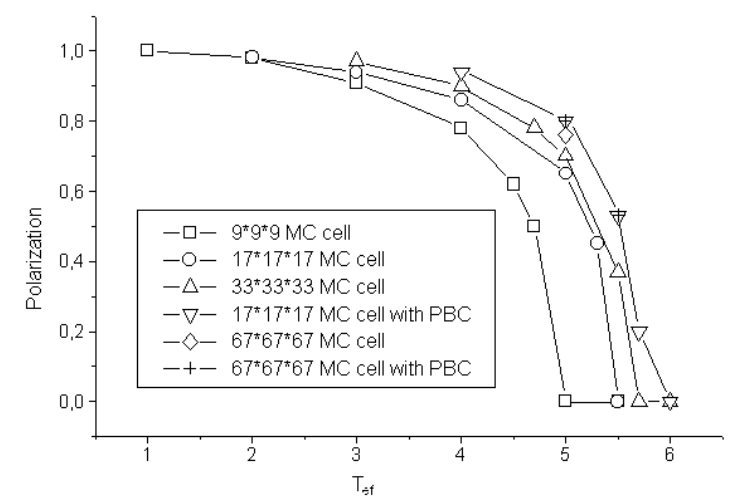

Figure 12. The results of MC calculations of polarization of a bulk sample of $\mathrm{Sn}_{2} \mathrm{P}_{2} \mathrm{~S}_{6}$ (MC cell with PBC) and of microscopic samples (MC cell with free boundary conditions) in the ANNNIadapted model.

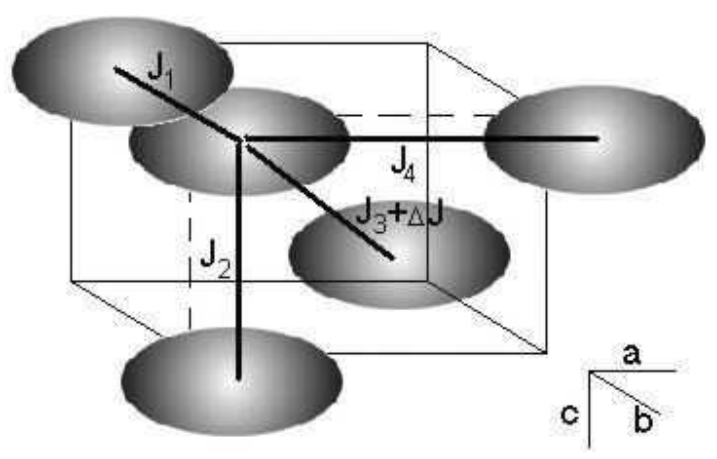

Figure 13. The scheme of crystal structure of $\mathrm{Sn}_{2} \mathrm{P}_{2} \mathrm{~S}_{6}$ in ANNNI-dipole model. the coefficient of interaction with the 
nearest adjacent pseudo-dipole $J_{3}$ is entered. Hereinafter, we shall call such a model the ANNNI-dipole model.

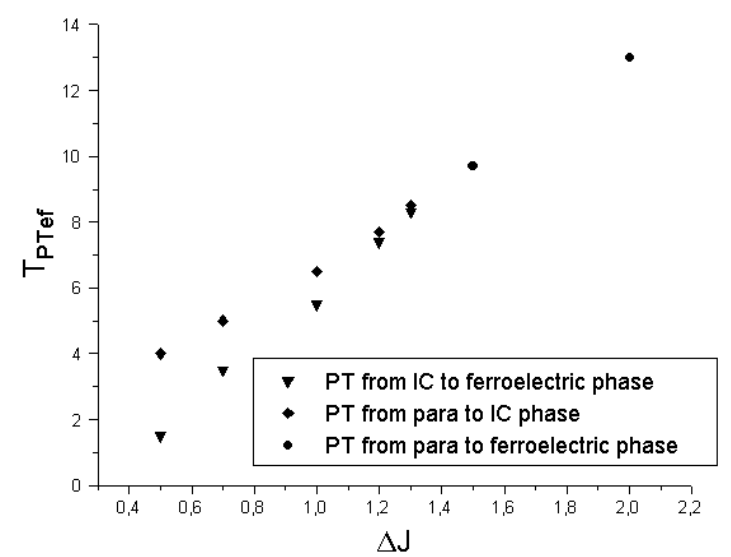

Figure 14. The MC calculated phase diagram of the ANNNI-dipole model in the area of the Lifshitz point.

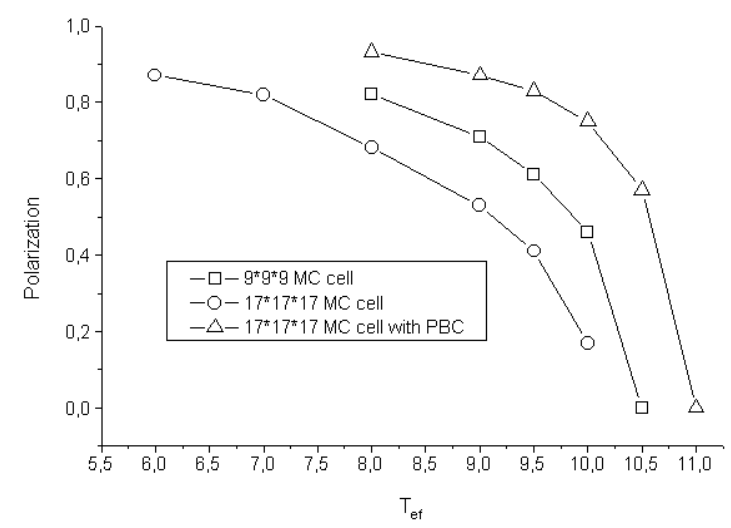

Figure 15. The results of MC calculations of polarization temperature dependence for the bulk sample of $\mathrm{Sn}_{2} \mathrm{P}_{2} \mathrm{~S}_{6}$ (MC cell with $\mathrm{PBC}$ ) and of microscopic samples (MC cell with free boundary conditions) in ANNNI-dipole model.

In this model there is only one correctional parameter $-\Delta J$. To study its effect on the phase diagram of the given model, calculations were carried out by the Monte Carlo method, the results of which are shown in figure 14. In this diagram we can see the same three phases i.e., ferroelectric, incommensurate and paraelectric one, as well as the Lifshitz point.

Applying the given model to the $\mathrm{Sn}_{2} \mathrm{P}_{2} \mathrm{~S}_{6}$ crystal is a more complex problem than for the previous ANNNIadapted model, because at transition from $\mathrm{Sn}_{2} \mathrm{P}_{2} \mathrm{~S}_{6}$ to $\mathrm{Sn}_{2} \mathrm{P}_{2} \mathrm{Se}_{6}$ lattice all parameters change. Now, we shall restrict ourselves to a qualitative analysis of a size effect for microcrystals far from the Lifshitz point (for $\Delta J=1.7$ ). In figure 15 , the results of these calculations for MC cells of different dimensions with periodic and free boundary conditions are shown.

The character of the size effect accords with the one found in the above mentioned before ANNNI-adapted model and qualitatively satisfies the experimentally detected one. The proposed ANNNI-dipole model, among the considered claims for a role of the most universal model, approaches the simulation of both bulk and microscopic samples of $\mathrm{Sn}_{2} \mathrm{P}_{2} \mathrm{~S}_{6}$ and qualitatively describes the size effect. Obviously, interesting experimental properties of $\mathrm{Sn}_{2} \mathrm{P}_{2} \mathrm{~S}(\mathrm{Se})_{6}$ ferroelectrics could be fully enough analyzed in this model. Similar phenomena are characteristic of different ferroelectrics that obviously make the considered models quite common and useful.

\section{Acknowledgements}

We appreciate Prof. R. Levitskii's stimulating discussions and his support at the start of MC simulations for the models investigated. 


\section{References}

1. Armstrong R.L. // Progress in NMR Spectroscopy, 1989, vol. 21, p. 151.

2. Giddy A.P., Dove M.T., Heine V. // J. Phys.: Condens. Matter, 1989, vol. 1, p. 8327.

3. Radescu S., Etxebarria I., Perez-Mato J.M. // J. Phys.: Condens. Matter, 1995, vol. 7, p. 585; Perez-Mato J.M., Etxebarria I., Radescu S., Ivantchev S. // Eur. Phys. J. B, 1999, vol. 12, p. 331.

4. Selke W., Fisher M.E. // Phys. Rev. B, 1979, vol. 20, p. 257; Selke W. // Physics Reports, 1988, vol. 170, p. 213; Neubert B., Pleimling M., Siems R. // Ferroelectrics, 1998, vol. 208-209, p. 141.

5. Vysochanskii Yu.M., Slivka V.Yu. // Sov. Phys. Usp., 1992, vol. 35, p. 123.

6. Vysochanskii Yu.M., Slivka V.Yu. Ferroelectrics of $\mathrm{Sn}_{2} \mathrm{P}_{2} \mathrm{~S}_{6}$ Family. Properties in the Vicinity of Lifshitz Point. Lviv, Oriana Nova, 1994 (in Russian).

7. Vysochanskii Yu.M., Maior M.M., Rizak V.M., Slivka V.Yu., Khoma M.M. // Sov. Phys. JETP, 1989, vol. 68, p. 782.

8. Khoma M.M., Molnar A.A., Vysochanskii Yu.M. // J. Phys. Stud., 1998, vol. 2, p. 524.

9. Hlinka J., Janssen T., Dvorak V. // J. Phys.: Condens. Matter, 1999, vol. 11, p. 3209.

10. Vysochanskii Yu.M., Drobnich A.V. // Ferroelectrics, 2000, vol. 236, p. 198.

11. Cho Y.W., Choi S.K., Vysochanskii Yu.M. // J. Mater. Res., 2001, vol. 16, p. 3317.

12. Eijt S.W.H., Currat R., Lorentzo J.E., Saint-Gregoire P., Hennion B., Vysochanskii Yu.M. // Eur. Phys. J. B, 1998, vol. 5, p. 169.

13. Eijt S.W.H., Currat R., Lorentzo J.E., Saint-Gregoire P., Katano S., Janssen T., Hennion B., Vysochanskii Yu.M. // J. Phys.: Condens. Matter, 1998, vol. 10, p. 4811.

14. Moriya K., Kuniyoshi H., Tashita K., Ozaki Y., Yano S., Matsuo T. // J. Phys. Soc. Jpn., 1998, vol. 67, p. 3505.

15. Baltrunas D., Grabar A.A., Mazeika K., Vysochanskii Yu.M. // J. Phys.: Condens. Matter, 1999, vol. 11, p. 2983.

16. Scott B., Pressprich M., Willet R.D., Cleary D.A. // J. Solid State Chem., 1992, vol. 96 , p. 294.

17. Israel R., de Gelder R., Smits J.M.M., Beurskens P.T., S.W.H. Eijt, Rasing Th., van Kempen H., Maior M.M., Motrja S.F. // Z. Kristallographie, 1998, vol. 213, p. 34 .

18. Enjalbert R., Galy J., Vysochanskii Yu., Ouedraogo A., Saint-Gregoire P. // Eur. Phys. J. B, 1999, vol. 8, p. 169.

19. Van Raaij G.H.V., Rubtsov A.N., Janssen T. // Ferroelectrics, 2000, vol. 240, p. 163.

20. Rubtsov A.N., Janssen T. // Europhys. Lett., 2001, vol. 53, p. 216; Van Raaij G.H.V., Janssen T. // Ferroelectrics, 2001, vol. 250, p. 135.

21. Drobnich A., Vysochanskii Yu. // Condens. Matter Phys., 1998, vol. 1, p. 331.

22. Kowasaki T. // Progr. Theor. Phys., 1984, vol. 71, p. 246.

23. Berezovsky S.V., Korda V.Yu., Klepikov V.F. // Phys. Rev. B, 2001, vol. 64, p. 64103.

24. Hlinka J., Smirnov M.S., Janssen T. // Ferroelectrics, 2001, vol. 250, p. 123.

25. Drobnich A., Vysochanskii Yu. // Ferroelectrics, 1999, vol. 233, p. 14.

26. Pleimling M. // Phys. Rev. B, 2002, vol. 65, p. 184406. 


\section{Характер фазового переходу та мікроскопічні моделі для сегнетоелектриків $\mathbf{S n}_{2} \mathbf{P}_{2} \mathbf{S}(\mathrm{Se})_{6}$}

\section{Ю.Височанський, А.Дробніч}

Інститут фізики та хімії твердого тіла, Ужгородський національний університет, 88000 Ужгород, вул. Підгірна, 46

Отримано 30 серпня 2002 р.

В статті досліджена кореляція між особливостями фазової діаграми (наявність на діаграмі точки Ліфшиця та трикритичної точки) та кросовером характеру фазових переходів від типу зміщення до типу лад-безлад на прикладі конкретних об'єктів, а саме сегнетоелектриків родини $\mathrm{Sn}_{2} \mathrm{P}_{2} \mathrm{~S}_{6}$. Аналізуються результати комп'ютерного моделювання з використанням мікроскопічних моделей, що найбільш адекватно відображають дипольне впорядкування в цих кристалах. Розглядається ефект розмірної залежності температури сегнетоелектричного фазового переходу для мікрокристалів сполуки $\mathrm{Sn}_{2} \mathrm{P}_{2} \mathrm{~S}_{6}$.

Ключові слова: сегнетоелектрики, кросовер від типу зміщення до типу лад-безлад, точка Ліфшиця, неспівмірна фаза, мікроскопічні моделі, моделювання методом Монте-Карло

PACS: $64.60 . \mathrm{Cn}$ 
